UB-ECM-PF-06/44

January 2007

gr-qc/0701090

\title{
Cosmologies with variable parameters and dynamical cosmon: implications on the cosmic coincidence problem
}

\author{
Javier Grande ${ }^{1}$, Joan Solà ${ }^{1,2}$ and Hrvoje Štefančićc $\check{c}^{1,3}$ \\ ${ }^{1}$ High Energy Physics Group, Dept. ECM, Universitat de Barcelona, \\ Diagonal 647, 08028 Barcelona, Catalonia, Spain \\ ${ }^{2}$ C.E.R. for Astrophysics, Particle Physics and Cosmologyt Barcelona \\ 3 Theoretical Physics Division, Rudjer Bošković Institute, P.O.B. 180, \\ HR-10002 Zagreb, Croatia \\ E-mail: jgrande@ecm.ub.es, sola@ifae.es and shrvoje@thphys.irb.hr
}

\begin{abstract}
Dynamical dark energy (DE) has been proposed to explain various aspects of the cosmological constant (CC) problem(s). For example, it is very difficult to accept that a strictly constant $\Lambda$-term constitutes the ultimate explanation for the DE in our Universe. It is also hard to acquiesce in the idea that we accidentally happen to live in an epoch where the CC contributes an energy density value $\rho_{\Lambda}=\Lambda / 8 \pi G$ right in the ballpark of the rapidly diluting matter density $\rho_{\mathrm{m}} \sim 1 / a^{3}$. It should perhaps be more plausible to conceive that the vacuum energy, $\rho_{\Lambda}$, is actually a dynamical quantity as the Universe itself. More generally, we could even entertain the possibility that the total $\mathrm{DE}$ is in fact a mixture of $\rho_{\Lambda}$ and other dynamical components (e.g. fields, higher order terms in the effective action etc) which can be represented collectively by an effective entity $X$ (dubbed the "cosmon"). The "cosmon", therefore, acts as a dynamical DE component different from the vacuum energy. While it can actually behave phantom-like by itself, the overall DE fluid may effectively appear as standard quintessence, or even mimic at present an almost exact CC behavior. Thanks to the versatility of such cosmic fluid we can show that a composite DE system of this sort (" $\Lambda \mathrm{XCDM")} \mathrm{may} \mathrm{have} \mathrm{a} \mathrm{key} \mathrm{to} \mathrm{resolving} \mathrm{the} \mathrm{mysterious} \mathrm{coincidence} \mathrm{problem.}$
\end{abstract}

\section{Introduction}

Modern Cosmology has reached a status of a mature empirical science. It is still far away from the level of precision of Particle Physics, but it is on the way. Independent data sets derived from the observation of distant supernovae [1], the temperature anisotropies of the CMB [2], the integrated Sachs-Wolfe effect [3], the lensing corrections on the propagation of light through weak gravitational fields [4], and the inventory of cosmic matter from the large scale structures (LSS) of the Universe [5] indicate altogether 
that our Universe is presently under a phase of accelerated expansion. It is of course tempting to simplify this state of affairs by just resorting to the existence of an absolutely constant (time-independent) $\mathrm{CC}$ term, $\Lambda$, in Einstein's equations. It is no less tempting to supersede this hypothesis with another - radically different- one: viz. to introduce a slowly evolving scalar field $\phi$ ("quintessence") whose potential, $V(\phi) \gtrsim 0$, accounts for the present value of the DE and whose equation of state (EOS) parameter $\omega_{\phi}=p_{\phi} / \rho_{\phi} \simeq-1+\dot{\phi}^{2} / V(\phi)$ is only slightly larger than -1 (hence insuring a negative pressure mimicking the $\Lambda$ case). In this way the DE can be a dynamical quantity taking different values throughout the history of the Universe. However, this possibility can not easily explain why the present value of the DE is so close to the rapidly decaying matter density, $\rho_{\mathrm{m}} \sim 1 / a^{3}$ - the so-called "cosmic coincidence problem". And even if it could (as some modified quintessence models propose [6]), there is after all a vacuum energy associated to the other fields (e.g. the electroweak Standard Model ones) and, therefore, such hypothetical scalar field cannot be the only one responsible for the vacuum energy. At the end of the day it does not seem to be such a wonderful idea to invent a field $\phi$ and simply replace $\rho_{\Lambda}$ with $\rho_{\phi} \simeq V(\phi)$. More fundamentally, $\Lambda$ could instead be conceived as a "running parameter" in QFT in curved space-time, as proposed in [7]. Here we go a bit beyond and suggest that the DE could involve, apart from a dynamical $\Lambda$, another collective component, " $X$ ", which does not necessarily represent any ad hoc field. It may stand for higher order terms in the effective action, perhaps in combination with some low-energy "relics" (e.g. a dilaton) from string theory, but in any case without being a full substitute for $\Lambda$. Of course we have to assume that the corresponding energy densities $\rho_{\mathrm{X}}$ and $\rho_{\Lambda}$ conspire so as to generate the tiny value of the DE density at present - the "old CC problem" [8]. While we cannot solve this problem at this stage, the dynamical nature of $\Lambda$ and $X$ gives at least allowance for this possibility to occur.

Here we focus on the second CC problem, the "coincidence problem" [8]. As we shall see, in the present framework we can provide a novel clue for a possible resolution of this problem. To start with, we note that once we impose the Bianchi identity in Einstein's equations (derived from the full effective action) it acts as a kind of "superselection energy sum rule" whereby the many terms on the r.h.s of these "effective" Einstein's equations must add up themselves to satisfy a local energy conservation law, irrespective of the inner details of the particular model. One of these terms is of course the vacuum energy, $\rho_{\Lambda}$, and the other terms can be treated as the aforesaid single effective entity " $X$ ", which we will refer to sometimes as the "cosmon". For obvious reasons we call this class of composite $(\Lambda, X)$-dark energy models the " $\Lambda$ XCDM models" [9, 10]. In them we still have some freedom in the way matter, vacuum and cosmon energy densities realize the local conservation law. Here we will explore just two possibilities that we call "type I" and "type II" $\Lambda$ XCDM models. In type I models matter is conserved and the total dynamical DE $\left(\rho_{\mathrm{D}}=\rho_{\Lambda}+\rho_{\mathrm{X}}\right)$ too. In type II models, instead, matter and cosmon densities are separately conserved, but the $\Lambda$ variation is compensated for by a variable gravitational coupling $G$. In the following we expand on these two possibilities and show that any of them could efficiently solve the coincidence problem. 


\section{Composite dark energy models}

For a DE medium consisting of several fluids with $p_{i}=\omega_{i} \rho_{i}(i=1,2, \ldots, n)$, the effective EOS parameter of the mixture reads:

$$
\omega_{\mathrm{e}}=\frac{p_{\mathrm{D}}}{\rho_{\mathrm{D}}}=\frac{\omega_{1} \rho_{1}+\omega_{2} \rho_{2}+\ldots}{\rho_{1}+\rho_{2}+\ldots},
$$

being in general a function of time or the (cosmological) redshift $z$, even if all $\omega_{i}$ are constant. Assuming a flat FLRW metric, Einstein equations for such a model lead to:

$$
\begin{aligned}
& H^{2}=\frac{8 \pi G}{3}\left(\rho_{\mathrm{m}}+\rho_{1}+\rho_{2}+\ldots\right) \\
& \frac{\ddot{a}}{a}=-\frac{4 \pi G}{3}\left[\rho_{\mathrm{m}}\left(1+3 \omega_{\mathrm{m}}\right)+\rho_{1}\left(1+3 \omega_{1}\right)+\rho_{2}\left(1+3 \omega_{2}\right) \ldots\right],
\end{aligned}
$$

where $\rho_{\mathrm{m}}$ stands for the density of matter-radiation. Equation (2) entails the following "generalized cosmic sum rule" valid at any $z$ :

$\hat{\Omega}_{\mathrm{m}}(z)+\hat{\Omega}_{1}(z)+\hat{\Omega}_{2}(z)+\ldots=1 ; \quad \hat{\Omega}_{i}(z) \equiv \frac{\rho_{i}(z)}{\rho_{\mathrm{c}}(z)}=\frac{8 \pi G \rho_{i}(z)}{3 H^{2}(z)}(i=\mathrm{m}, 1,2, \ldots)$.

We denote $\hat{\Omega}_{i}(z=0) \equiv \Omega_{i}^{0}$. If the DE is a single fluid, the corresponding sum rule at present $\left(\Omega_{\mathrm{m}}^{0}+\Omega_{\mathrm{D}}^{0}=1\right)$ enforces $\rho_{\mathrm{D}}^{0}$ to be positive, given that $\Omega_{\mathrm{m}}^{0} \simeq 0.3$ [1] . If in addition the DE is self-conserved, then necessarily $\rho_{\mathrm{D}}>0$ at any time. On the other hand, for a composite DE it is clear that (4) could be fulfilled even if one or more of the DE components have negative energy density, and then many possibilities open up. For instance, from (3) we note that a component with $\omega_{i}<-1 / 3$ and $\rho_{i}<0$ would decelerate the expansion instead of accelerating it (cf. Fig.(1). In particular, phantom-like $\left(\omega_{i}<-1\right)$ components with negative energy density $\left(\rho_{i}<0\right)$ also observe the strong energy condition (SEC, cf. Fig.1b) just the same as matter. In contrast, the "standard" phantom components $\left(\omega_{i}<-1, \rho_{i}>0\right)$ not only violate all the classical energy conditions but also may produce an specially acute anti-gravitational effect which eventually leads to a kind of singularity known as "Big Rip" [11] whereby all bound systems, without exception, are eventually ripped off and hence destroyed. At variance with these ugly prospects, phantom components with $\rho_{i}<0$ cause a fast deceleration that may result into the future stopping and subsequent reversal of the Universe expansion. This is rather significant since, as we will see, the stopping of the expansion can be linked to the solution of the coincidence problem. Thus, phantom components with negative energy density (hence positive pressure) behave as a sort of "unclustering matter" pervading the Universe; it has ben called "Phantom matter" (cf. PM region in Fig. (1) [9]. The aforementioned "cosmon", for instance, can behave as PM.

\section{3. $\Lambda \mathrm{XCDM}$ cosmologies}

As a simple realization of the idea of a composite DE we have considered a dual DE system consisting of a running $\Lambda$ in potential interaction with another dynamical entity $X$ (which we have referred to as the "cosmon" - see [12] for the origin of the name). 

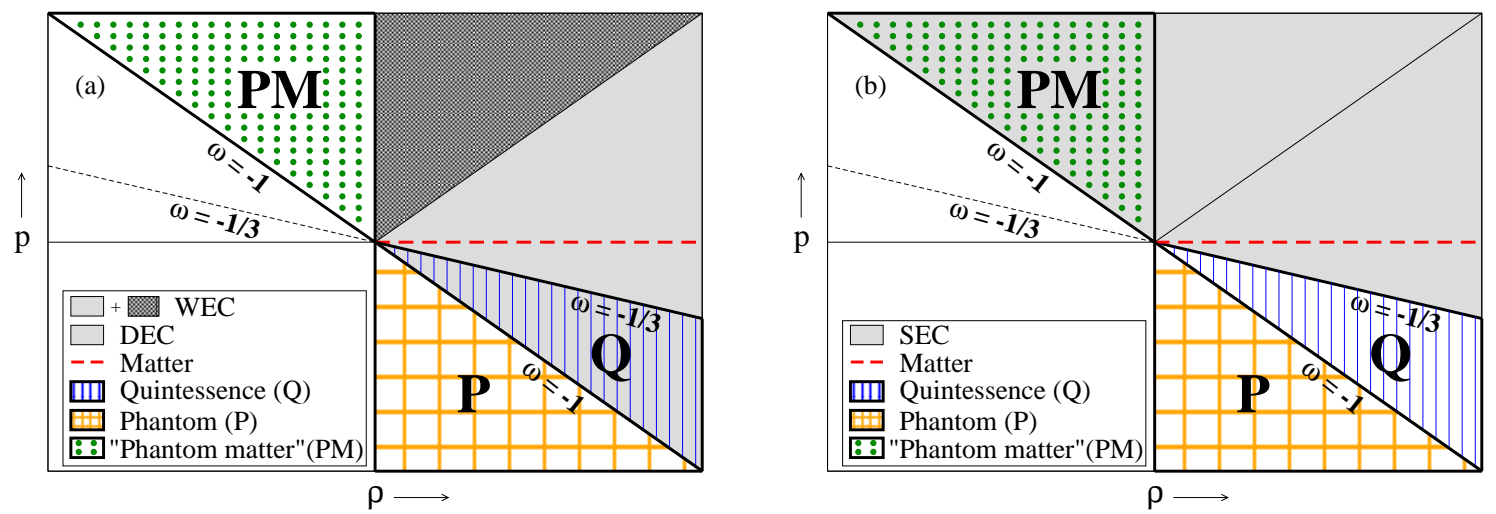

Figure 1. (a) The Weak (WEC, $\rho \geq 0$ and $\rho+p \geq 0$ ) and Dominant (DEC, $\rho \geq|p|$ ) energy conditions. The EOS regions of matter, quintessence (Q) and "standard" phantom $(\mathrm{P})$ are shown, together with the "phantom matter" $(\mathrm{PM})$ region $\left(\omega_{\mathrm{e}}<-1\right.$ with $\rho<0$ ); (b) The Strong energy condition (SEC, $\rho+p \geq 0$ and $\rho+3 p \geq 0$ ) insuring attractive gravitation force, is only satisfied by matter and PM.

As explained in Section 1, the $X$ component would stand for any contribution to the DE energy other than the vacuum energy effects. We call this scenario the $\Lambda$ XCDM model [9]. The evolution of $\Lambda$ is (as any parameter in QFT) tied to the renormalization group (RG) in curved space-time [7]. The running equation we will consider here for the $\mathrm{CC}$ density is one that has been thoroughly studied in the literature [7, 13]:

$$
\frac{\mathrm{d} \rho_{\Lambda}}{\mathrm{d} \ln \mu}=\frac{\sigma}{(4 \pi)^{2}} M^{2} \mu^{2} \equiv \frac{3 \nu}{4 \pi} M_{\mathrm{P}}^{2} \mu^{2} .
$$

Here $\mu$ is the energy scale associated to the RG in Cosmology (that we will identify with the Hubble function, i.e. $\mu=H$ at any epoch, see [13] $)$ and $\nu=(\sigma / 12 \pi) M^{2} / M_{\mathrm{P}}^{2}$ is a free parameter, $M$ being the effective mass of the heavy particles contributing to the $\beta$-function of the CC and $\sigma= \pm 1$ depending on whether bosons or fermions dominate. If $M=M_{\mathrm{P}}$ (Planck mass), $|\nu|$ takes the value $\nu_{0} \equiv 1 / 12 \pi \simeq 0.026$. Thus, we naturally expect $|\nu| \ll 1$. Introducing an specific model for $\Lambda$ allows us to preserve the generality of the cosmon $X$. Let us only mention that it could e.g. be some scalar field $\chi$ resulting from low-energy string theory (e.g. a pseudo-dilaton, as in the original paper [12]), or account for the effective behavior of a mixture of dynamical fields of various sorts and/or higher order curvature terms in the action. We really do not need to specify its ultimate nature here because, as we shall see, the kind of cosmological implications that we will investigate (in particular its impact on the coincidence problem ) do not depend on it.

We start the formulation of the model from the most general form of the Bianchi identity on both sides of Einstein's equations: $\nabla^{\nu} G_{\mu \nu}=8 \pi \nabla^{\nu}\left(G T_{\mu \nu}\right)=0$, where $T_{\mu \nu}$ is the effective energy-momentum tensor including all the terms on the r.h.s of Einstein's equations. Assuming a FLRW metric and describing $T_{\mu \nu}$ as a mixture of fluids (including 
matter), the $\mu=0$ component of the Bianchi identity above yields:

$$
\frac{\mathrm{d}}{\mathrm{d} t}\left[G\left(\sum_{i} \rho_{i}\right)\right]+G H \sum_{i} \alpha_{i} \rho_{i}=0, \quad \alpha_{i} \equiv 3\left(1+\omega_{i}\right),
$$

where the various $\rho_{i}$ stand for the energy densities of the different fluids. In our case we have matter-radiation and a composite DE:

$$
\rho_{\mathrm{m}}=\rho_{\mathrm{M}}+\rho_{\mathrm{R}}, \quad \rho_{\mathrm{D}}=\rho_{\mathrm{X}}+\rho_{\Lambda},
$$

$\rho_{\mathrm{X}}(t)$ being the dynamical density of the cosmon and $\rho_{\Lambda}(t)$ the energy density of the running $\Lambda$. In principle we also admit the possibility of having a variable Newton's coupling, $G=G(t)$. Note that this time variability of the cosmological parameters is consistent with the Cosmological Principle and that the general covariance of the theory is insured by the fulfilment of the general Bianchi identity (6) . The two DE components have EOS parameters $\omega_{\Lambda}=-1$ and $\omega_{\mathrm{X}}$ respectively, with:

$$
-1-\delta / 3<\omega_{\mathrm{X}}<-1 / 3 \Longrightarrow-\delta<\alpha_{\mathrm{X}}<2<\alpha_{\mathrm{m}} \quad(\delta \gtrsim 0)
$$

i.e. $X$ can be quintessence or phantom-like. We consider two possible realizations of the $\Lambda$ XCDM model, in both cases with local conservation of matter:

(i) Type I $\Lambda$ XCDM model: $\dot{G}=0$ and $\rho_{\mathrm{m}}$ conserved, hence $\rho_{\mathrm{D}}$ is also conserved.

(ii) Type II $\Lambda$ XCDM model: $\dot{G} \neq 0$ with $\rho_{\mathrm{m}}$ and $\rho_{\mathrm{X}}$ conserved.

Next we will explain the main features of these two models, showing that they can both alleviate the coincidence problem and match the available data. Comparatively, modified (interactive) quintessence models can tackle the coincidence problem only at the expense of matter non-conservation [6]. Herein we limit ourselves to the simplest case, in which spatial flatness and constant $\omega_{\mathrm{X}}$ are assumed, although more general results can be obtained analytically [9].

\section{Type I $\Lambda$ XCDM models}

In this case we assume that Newton's coupling $G$ is constant and matter-radiation is conserved, $\dot{\rho}_{\mathrm{m}}+\alpha_{\mathrm{m}} \rho_{\mathrm{m}} H=0$. These conditions in turn imply the conservation of the total DE density $\rho_{\mathrm{D}}$. The relevant set of equations are the Friedmann equation (2), the conservation of DE arising from the Bianchi identity (6) , and the RG model for $\Lambda$ (5):

$$
\begin{aligned}
& H^{2}=\frac{8 \pi G}{3}\left(\rho_{\mathrm{m}}+\rho_{\Lambda}+\rho_{\mathrm{X}}\right), \\
& \dot{\rho}_{\mathrm{D}}+\alpha_{\mathrm{D}} \rho_{\mathrm{D}} H=\dot{\rho}_{\Lambda}+\dot{\rho}_{\mathrm{X}}+\alpha_{\mathrm{X}} \rho_{\mathrm{X}} H=0, \\
& \frac{\mathrm{d} \rho_{\Lambda}}{\mathrm{d} \ln H}=\frac{3 \nu}{4 \pi} M_{\mathrm{P}}^{2} H^{2} .
\end{aligned}
$$

These equations can be rewritten as an autonomous system in terms of the new independent variable $\zeta=-\ln (1+z)$ (the asymptotic past and future lying at $\zeta=-\infty$ 
and $\zeta=\infty$ respectively and the present at $\zeta=z=0)$ :

$$
\begin{aligned}
& \Omega_{\mathrm{X}}^{\prime}=-\left[\nu \alpha_{\mathrm{m}}+(1-\nu) \alpha_{\mathrm{X}}\right] \Omega_{\mathrm{X}}-\nu \alpha_{\mathrm{m}} \Omega_{\Lambda}+\nu \alpha_{\mathrm{m}} \Omega_{\mathrm{c}}, \\
& \Omega_{\Lambda}^{\prime}=\nu\left(\alpha_{\mathrm{m}}-\alpha_{\mathrm{X}}\right) \Omega_{\mathrm{X}}+\nu \alpha_{\mathrm{m}} \Omega_{\Lambda}-\nu \alpha_{\mathrm{m}} \Omega_{\mathrm{c}}, \\
& \Omega_{\mathrm{c}}^{\prime}=\left(\alpha_{\mathrm{m}}-\alpha_{\mathrm{X}}\right) \Omega_{\mathrm{X}}+\alpha_{\mathrm{m}} \Omega_{\Lambda}-\alpha_{\mathrm{m}} \Omega_{\mathrm{c}}
\end{aligned}
$$

where ${ }^{\prime} \equiv \mathrm{d} / \mathrm{d} \zeta$. In the previous system the density fractions $\Omega_{i}$ are normalized with respect to the present critical energy density (in contrast to the $\hat{\Omega}_{i}$ in (44)):

$$
\Omega_{i}(z)=\frac{\rho_{i}(z)}{\rho_{\mathrm{c}}^{0}}=\frac{8 \pi G \rho_{i}(z)}{3 H_{0}^{2}} \quad(i=\mathrm{X}, \Lambda, \mathrm{c}) .
$$

Clearly, $\Omega_{i}(z) / \hat{\Omega}_{i}(z)=H^{2}(z) / H_{0}^{2}=\Omega_{c}(z)$. The solution of (12) reads as follows:

$$
\boldsymbol{\Omega}(\zeta) \equiv\left(\Omega_{\mathrm{X}}(\zeta), \Omega_{\Lambda}(\zeta), \Omega_{\mathrm{c}}(\zeta)\right)=C_{1} \mathbf{v}_{\mathbf{1}} e^{\lambda_{1} \zeta}+C_{2} \mathbf{v}_{\mathbf{2}} e^{\lambda_{2} \zeta}+C_{3} \mathbf{v}_{\mathbf{3}}
$$

with:

$$
\begin{array}{lll}
\lambda_{1}=-\alpha_{\mathrm{X}}(1-\nu), & \lambda_{2}=-\alpha_{\mathrm{m}}, & \lambda_{3}=0 . \\
\mathbf{v}_{\mathbf{1}}=(1-\nu, \nu, 1), & \mathbf{v}_{\mathbf{2}}=\left(\frac{-\nu \alpha_{\mathrm{m}}}{\alpha_{\mathrm{m}}-\alpha_{\mathrm{X}}}, \nu, 1\right), & \mathbf{v}_{\mathbf{3}}=(0,1,1) . \\
C_{1}=1-C_{2}-C_{3}, & C_{2}=\frac{\Omega_{\mathrm{m}}^{0}\left(\alpha_{\mathrm{m}}-\alpha_{\mathrm{X}}\right)}{\alpha_{\mathrm{m}}-\alpha_{\mathrm{X}}(1-\nu)}, & C_{3}=\frac{\Omega_{\Lambda}^{0}-\nu}{1-\nu} .
\end{array}
$$

The coefficients $C_{k}$ result from the boundary conditions at present: $\Omega_{i}(0)=\Omega_{i}^{0}=\hat{\Omega}_{i}(0)$. Assuming the aforementioned prior $\Omega_{\mathrm{m}}^{0} \simeq 0.3$, our model contains three free parameters: $\nu, \omega_{\mathrm{X}}$ and $\Omega_{\Lambda}^{0}=\Omega_{\mathrm{D}}^{0}-\Omega_{\mathrm{X}}^{0} \simeq 0.7-\Omega_{\mathrm{X}}^{0}$.

\subsection{Nucleosynthesis and the coincidence problem}

As the expansion rate is sensitive to the amount of DE, we must ensure that our model does not spoil the predictions of primordial nucleosynthesis. Thus, we will ask the ratio between DE and matter radiation densities,

$$
r(z) \equiv \frac{\rho_{\mathrm{D}}(z)}{\rho_{\mathrm{m}}(z)}
$$

to be small enough at the nucleosynthesis epoch, say $\left|r_{\mathrm{N}} \equiv r\left(z=z_{\mathrm{N}} \sim 10^{9}\right)\right| \lesssim 10 \%$ ([13, 14], see also [15]). From the solution (14) and after a straightforward calculation we find that:

$$
\left|r_{\mathrm{N}}\right|<10 \% \Longleftrightarrow \frac{|\epsilon|}{\omega_{\mathrm{R}}-\omega_{\mathrm{X}}+\epsilon} \simeq|\epsilon|<0.1, \quad \epsilon \equiv \nu\left(1+\omega_{\mathrm{X}}\right)
$$

where $\omega_{\mathrm{R}}=1 / 3$ is the EOS parameter of radiation. Note that only for $\nu=0$ would the DE density be vanishing at the nucleosynthesis time; this shows that, in general, in the type I $\Lambda$ XCDM model the presence of DE takes place at all epochs of the evolution. Looking again at (17) but this time at the matter dominated era, one can show that $r$ can have -at most- one extremum at some $z=z_{\mathrm{e}}$ in the future [9]. We are interested in the case in which this extremum is a maximum, since this will help solving the cosmic coincidence problem. Indeed, if $r$ remains bounded from above -and maybe even of 
order 1 - for the entire Universe lifetime, the fact that $r_{0} \equiv r(z=0) \sim 1$ would no longer look like a coincidence. The conditions for the extremum to exist and to be a maximum are shown to be [9]:

$\frac{\Omega_{\Lambda}^{0}-\nu}{\omega_{\mathrm{X}}\left(\Omega_{\mathrm{X}}^{0}+\nu \Omega_{\mathrm{M}}^{0}\right)-\epsilon\left(1-\Omega_{\Lambda}^{0}\right)}>0 \quad$ and $\quad \alpha_{\mathrm{X}}\left(\Omega_{\Lambda}^{0}-\nu\right)<0$.

Remarkably, the existence of a maximum for $r(z)$ entails a halt of the cosmic expansion at some future point $z=z_{\mathrm{s}}$ (stopping redshift). To prove this, let us notice that Einstein's equations (9) and (3) for the type I $\Lambda$ XCDM model imply:

$$
\begin{aligned}
& \lim _{z \rightarrow-1} H^{2} / H_{0}^{2}=\lim _{z \rightarrow-1} \Omega_{\mathrm{D}}, \\
& \left.\frac{\ddot{a}}{a}\right|_{t=t_{0}}=-\frac{4 \pi G}{3}\left[\left(1+r_{0}\right)+r^{\prime}(0)\right],
\end{aligned}
$$

where $z \rightarrow-1$ stands for the remote future, $t_{0}$ is the present time and $r^{\prime}(z)=\mathrm{d} r(z) / \mathrm{d} z$. Since $r_{0}>0$, the current state of accelerated expansion requires $r^{\prime}(z=0)<0$ (i.e. $r$ is presently increasing with time: $\dot{r}\left(t=t_{0}\right)>0$ ). Now, if the r.h.s of (20) is positive, $\lim _{z \rightarrow-1} r(z)=\infty$ and the ratio is unbounded. Moreover, knowing that the function $r=r(z)$ can have at most one extremum [9] the foregoing conditions imply that there cannot be any extremum in the future. Thus, in this case the DE cannot become negative to stop the expansion. If, instead, the r.h.s of (20) is negative, then $H\left(z_{\mathrm{s}}\right)=0$ at some $z_{\mathrm{s}}>-1$. However, being $r$ presently positive and increasing with time, this situation can only be compatible with $\lim _{z \rightarrow-1} r(z)=-\infty$ if there is a maximum of $r$ at some point between $z=0$ and $z=z_{\mathrm{s}}$ (q.e.d.). Note that the condition $r^{\prime}(0)<0$ and the uniqueness of the extremum enforce the maximum to occur always in the future.

\subsection{Some possible scenarios}

Within the type I $\Lambda$ XCDM model there are many scenarios compatible with stopping (and subsequent reversal) of the expansion in the future. As shown in the last section, this implies the existence of a maximum of the ratio $r$ with the consequent alleviation of the coincidence problem. A very convenient form to identify these scenarios is by studying the phase trajectories of the autonomous system (12). Notice that an asymptotically negative value of the third component of (14) $), \Omega_{\mathrm{c}}(z) \equiv H^{2}(z) / H_{0}^{2}$, would indicate the existence of a stopping point. Let us discuss here just two representative cases - for a comprehensive analysis see [9]:

- 1) $-\delta<\alpha_{\mathrm{X}}<0$ (phantom-like cosmon) and $\nu<1$. Looking at the eigenvalues (15), we see that $\lambda_{1}>0, \lambda_{2}<0$, so there is a saddle point in the phase space:

$$
\Omega^{*}=\left(0, \Omega_{\Lambda}^{0}, \Omega_{\Lambda}^{0}\right)
$$

from which all trajectories diverge with the evolution (as $\zeta \rightarrow \infty$ ). However, if $C_{1}<0$ in (15), $\Omega_{\mathrm{c}}(z \rightarrow-1)<0$, implying the stopping of the expansion as discussed above. Using (18), the stopping condition acquires the form:

$$
C_{1}=1-C_{2}-C_{3}=\frac{1-\Omega_{\Lambda}^{0}}{1-\nu}-\frac{\Omega_{\mathrm{M}}^{0}\left(\omega_{\mathrm{m}}-\omega_{\mathrm{X}}\right)}{\omega_{\mathrm{m}}-\omega_{\mathrm{X}}+\epsilon} \simeq \frac{1-\Omega_{\Lambda}^{0}}{1-\nu}-\Omega_{\mathrm{M}}^{0}<0 .
$$



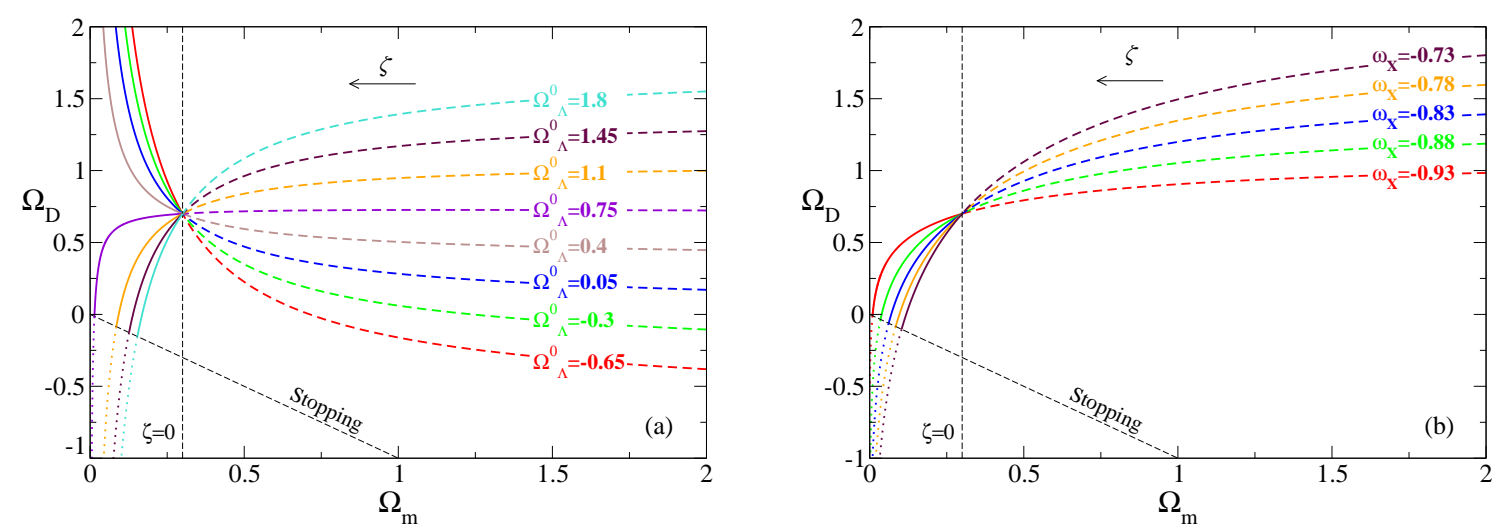

Figure 2. Phase trajectories of the autonomous system (12) in the $\left(\Omega_{\mathrm{m}}, \Omega_{\mathrm{D}}\right)$ plane for different values of the parameters. Dashed lines show the parts of the curves corresponding to our past, full lines the parts between the present moment and the stopping (if there is stopping) and dotted lines the inaccessible part of the trajectory after the stopping; (a) $\omega_{\mathrm{X}}=-1.85, \nu=-\nu_{0}$ (cf. case 1 in the text) and different choices of $\Omega_{\Lambda}^{0}$ (b) $\Omega_{\Lambda}^{0}=-2, \nu=0.96$ (cf. case 2) and different values of $\omega_{\mathrm{X}}$.

We can check that this relation insures: a) the fulfilment of the conditions (19) for the existence of a maximum; b) $\Omega_{\mathrm{X}}^{0}<-\nu \Omega_{\mathrm{M}}^{0}$. Thus for $0<\nu<1$ the cosmon behaves as PM, whilst for $\nu<0$ behaves as a "conventional" phantom.

- 2) $0<\alpha_{\mathrm{X}}<2$ (quintessence-like cosmon) and $\nu<1$. Now the non-vanishing eigenvalues are both negative $\lambda_{1}<0, \lambda_{2}<0$. Therefore, there is a ( $\nu$-dependent) node towards which all phase trajectories are attracted, namely

$$
\Omega^{*}=\left(0, \frac{\Omega_{\Lambda}^{0}-\nu}{1-\nu}, \frac{\Omega_{\Lambda}^{0}-\nu}{1-\nu}\right) .
$$

This time, the attraction towards the node will be stopped for the curves satisfying:

$$
\frac{\Omega_{\Lambda}^{0}-\nu}{1-\nu}<0 \quad \Rightarrow \quad \Omega_{\Lambda}^{0}<\nu<1 .
$$

The projections onto the $\left(\Omega_{\mathrm{m}}, \Omega_{\mathrm{D}}\right)$ plane of the phase trajectories for these two scenarios have been plotted in Fig. $2 a, b$. They show respectively the existence of a saddle point or a node and the stopping of the curves that fulfil (23) or (25). Note that the solution of the coincidence problem can take place in both scenarios even for the simplest situation, namely for $\nu=0$. In this case, the DE of the model is just the sum of a self-conserved cosmon and a strictly constant $\Lambda$ :

$$
\Omega_{\mathrm{D}}(z)=\Omega_{\Lambda}^{0}+\Omega_{\mathrm{X}}^{0}(1+z)^{\alpha \mathrm{X}},
$$

and the stopping conditions deduced above just read: $\Omega_{\mathrm{X}}^{0}<0$ (case 1 ) and $\Omega_{\Lambda}^{0}<0$ (case 2 ), corresponding to a Universe containing phantom matter or a negative $\Lambda$ respectively. In both cases the overall effective EOS of the DE is quintessence-like. Even though $\nu=0$ does the job as far as the cosmic coincidence problem is concerned, the possibility to solve that problem also for $\nu \neq 0$ allows us to modulate the effective EOS behavior of the model and describe features that can be potentially observed in it (see next section). 

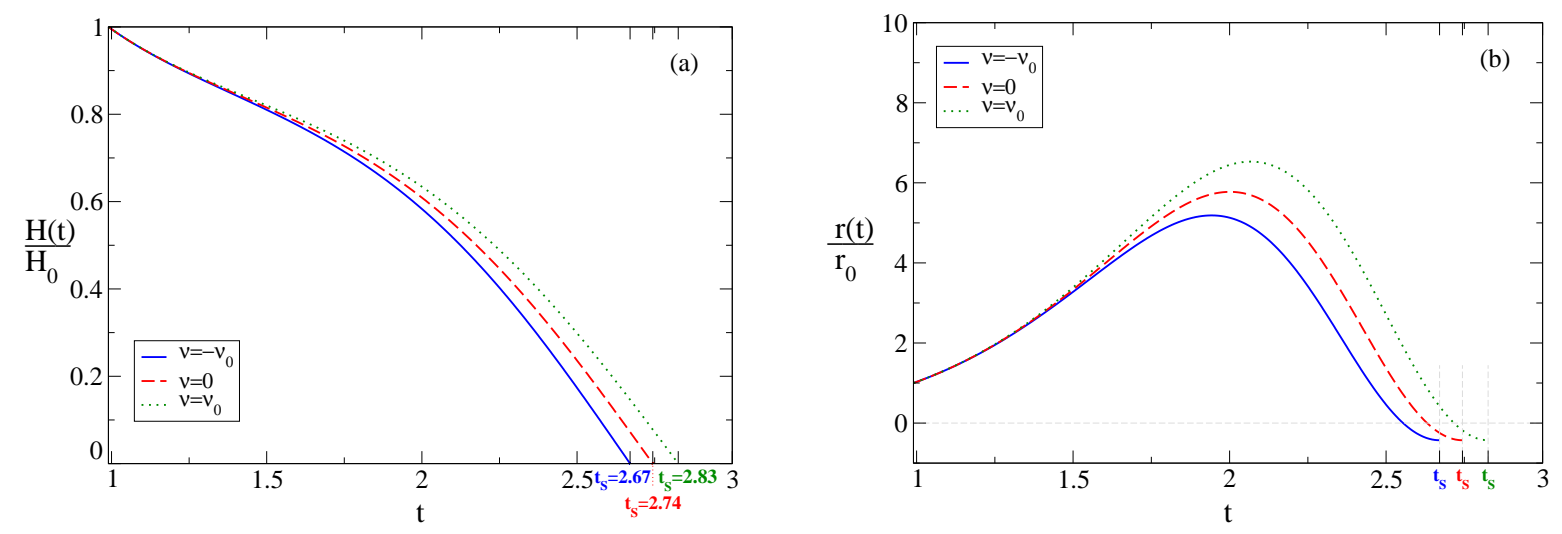

Figure 3. (a) The expansion rate (normalized to its present value $H_{0}$ ) as a function of the cosmic time, $t$, in the type I model for the following parameter values: $\omega_{\mathrm{X}}=-1.85$, $\Omega_{\Lambda}^{0}=0.75$ and $\nu=-\nu_{0}, 0,+\nu_{0}$, showing the existence of a stopping point; (b) The ratio $r=\rho_{\mathrm{D}} / \rho_{\mathrm{m}}$ (in units of its present value $r_{0}$ ) as a function of $t$, illustrating the presence of both a maximum and a stopping point in the future, what constitutes a possible explanation of the coincidence problem. The cosmic time $t$ is measured in Hubble time units $H_{0}^{-1}$; the present moment lies at $t \simeq 0.99$ (i.e. 13.7 Gyr).

\subsection{A numerical example}

Let us now illustrate the properties of the model through an specific example, namely $\omega_{\mathrm{X}}=-1.85, \Omega_{\Lambda}^{0}=0.75$ and different values of $\nu<1$. These values correspond to the first scenario discussed in the previous section. As long as we take $\nu$ sufficiently small, condition (23) will be satisfied and we should get both the stopping of the expansion and a maximum in the ratio $r$. In Fig. 3a, b we can observe these features for three different values of $\nu\left(0\right.$ and $\left.\pm \nu_{0}\right)$. There we have plotted the Hubble function and the ratio $r$ as functions of the cosmic time - which requires solving numerically the model. We see that the maximum and the stopping point take place far away in the future. In these cases, the halt of the expansion is caused by the behavior of the cosmon as PM rather than to a negative $\Lambda$, as we can appreciate in Fig. 4h. In this plot we also notice that the signs of the two components of the DE (which are individually unobservable) can change during the evolution.

Of course, in order to claim that our model provides a solution, or at least an important alleviation of the coincidence problem, we must be sure that this holds for a significant part of the full parameter space. We have confirmed this point through a comprehensive numerical sampling of it, and moreover we have found that the maximum of the ratio $r$ can stay rather small, say $1-10$ times its present value $r_{0}[9]$.

The comparison between a particular DE model and the observational data is made mainly through the EOS. The effective EOS of the type I $\Lambda \mathrm{XCDM}$ model is given by:

$$
\omega_{\mathrm{e}}^{(I)}=\frac{p_{\mathrm{D}}}{\rho_{\mathrm{D}}}=\frac{p_{\Lambda}+p_{\mathrm{X}}}{\rho_{\Lambda}+\rho_{\mathrm{X}}}=\frac{-\rho_{\Lambda}+\omega_{\mathrm{X}} \rho_{\mathrm{X}}}{\rho_{\Lambda}+\rho_{\mathrm{X}}}=-1+\left(1+\omega_{\mathrm{X}}\right) \frac{\rho_{\mathrm{X}}}{\rho_{\mathrm{D}}},
$$

and it can display a rich variety of behaviors while being in agreement with the most 

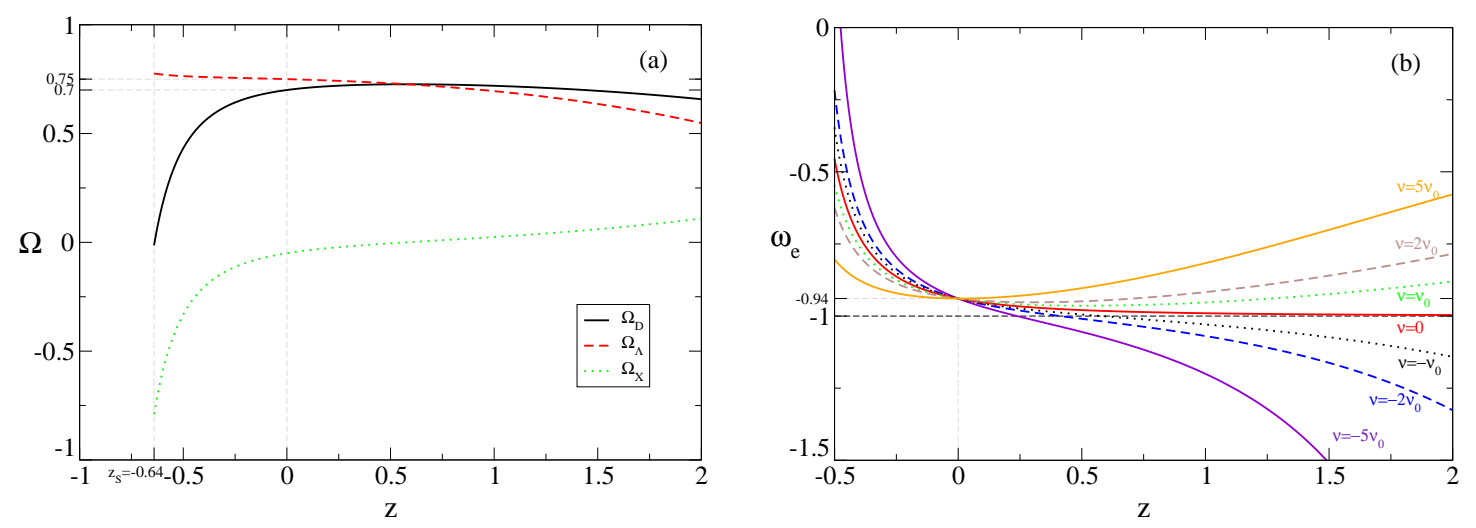

Figure 4. (a) The evolution of the density fraction $\Omega$ for the total DE and each one of its components, $X$ and $\Lambda$, in the type I model. The values of the parameters are the same as in Fig. 3 with the choice $\nu=-\nu_{0}$. We see that the stopping of the expansion (characterized by the value $\Omega_{\mathrm{D}}\left(z_{s}\right)=-\Omega_{\mathrm{m}}\left(z_{s}\right)$ ) is achieved thanks to $\Omega_{\mathrm{X}}$ being negative, i.e. to the behavior of the cosmon as PM; (b) Different behaviors of the EOS of the $\Lambda \mathrm{XCDM}$ model for the same $\omega_{\mathrm{X}}$ and $\Omega_{\Lambda}^{0}$ as in Figure 3 and different values of $\nu$.

recent data [2]. This is shown in Fig.4b, where we see that, depending on the value of $\nu$, the EOS can be quintessence-like, mimic (in some cases almost exactly) a pure CC term or even present a mild transition from the phantom to the quintessence regime.

\subsection{Asymptotic behavior in the past: a signature of the model}

Let us finally elaborate on a characteristic feature of type I models with running $\Lambda$ that could serve to distinguish them from other DE models. From the solution (14), we find that in the asymptotic past:

$$
\begin{array}{ll}
\Omega_{\mathrm{D}}(z \gg 1)=-\frac{\epsilon}{\omega_{\mathrm{m}}-\omega_{\mathrm{X}}+\epsilon} \Omega_{\mathrm{m}}^{0}(1+z)^{\alpha_{\mathrm{m}}}, & (\nu \neq 0) \\
\omega_{\mathrm{e}}^{(I)}(z \gg 1)=-1+\left(1+\omega_{\mathrm{X}}\right) \frac{\Omega_{\mathrm{X}}(z \gg 1)}{\Omega_{\mathrm{D}}(z \gg 1)} \rightarrow \omega_{\mathrm{m}}, & (\nu \neq 0) .
\end{array}
$$

Thus, surprisingly, at very high redshift the effective EOS of the DE coincides with that of matter-radiation: $\omega_{\mathrm{e}}^{(I)} \rightarrow \omega_{\mathrm{m}}$. Moreover, the Hubble function at high redshift reads:

$H^{2}(z \gg 1) \simeq H_{0}^{2} \Omega_{\mathrm{m}}^{0 *}(1+z)^{\alpha_{\mathrm{m}}}, \quad \Omega_{\mathrm{m}}^{0 *}=\Omega_{\mathrm{m}}^{0}\left(1-\frac{\epsilon}{\omega_{\mathrm{m}}-\omega_{\mathrm{X}}+\epsilon}\right)$,

which means that the value $\Omega_{\mathrm{m}}^{0 *}$ of the density fraction of matter inferred from very high $z$ data (e.g. from CMB) could differ from that obtained by low $z$ experiments $\left(\Omega_{\mathrm{m}}^{0}\right)$, typically from supernovae. The relative difference, $\left|\Omega_{\mathrm{m}}^{0 *}-\Omega_{\mathrm{m}}^{0}\right| / \Omega_{\mathrm{m}}^{0}$, is given just by the same expression as the nucleosynthesis constraint (18); therefore this effect could amount to a measurable $10 \%$ discrepancy and, if detected, would constitute a distinctive signature of type I $\Lambda$ XCDM models. 


\section{Type II $\Lambda$ XCDM models}

In this case we let the Newton coupling $G$ be variable and ask for the conservation of matter-radiation. These two conditions allow in turn the conservation of the $X$ component, $\dot{\rho}_{\mathrm{X}}+\alpha_{\mathrm{X}} \rho_{\mathrm{X}} H=0$. We adopt the same RG equation (5) as before for the running of $\Lambda$. With all this in mind, we obtain the set of equations defining the type II models:

$$
\begin{aligned}
& H^{2}=\frac{8 \pi G}{3}\left(\rho_{\mathrm{m}}+\rho_{\Lambda}+\rho_{\mathrm{X}}\right), \\
& \left(\rho_{\mathrm{m}}+\rho_{\Lambda}+\rho_{\mathrm{X}}\right) d G+G d \rho_{\Lambda}=0, \\
& \frac{\mathrm{d} \rho_{\Lambda}}{\mathrm{d} \ln \mu}=\frac{3 \nu}{4 \pi} M_{\mathrm{P}}^{2} \mu^{2} .
\end{aligned}
$$

This system can be analytically solved to determine $G$ as a function of the scale $\mu=H$, with the following result:

$$
G(H)=\frac{G_{0}}{1+\nu \ln \frac{H^{2}}{H_{0}^{2}}}
$$

where $G_{0}=1 / M_{\mathrm{P}}^{2}$. This equation suggests that $\nu$ acts also as the $\beta$-function for the RG running of $G$. We can also express $G=G(z)$ as an implicit function of the redshift $z$ :

$\frac{1}{g(z)}-1+\nu \ln \left(\frac{1}{g(z)}-\nu\right)=\nu \ln \left[\Omega_{\mathrm{m}}^{0}(1+z)^{\alpha_{\mathrm{m}}}+\Omega_{\mathrm{X}}^{0}(1+z)^{\alpha_{\mathrm{X}}}+\Omega_{\Lambda}^{0}-\nu\right]$,

where we have defined $g(z) \equiv G(z) / G_{0}$. Once this function is determined, $\rho_{\Lambda}=\rho_{\Lambda}(z)$ can also be derived:

$$
\rho_{\Lambda}(z)=\frac{\rho_{\Lambda}^{0}+\nu\left(\rho_{\mathrm{m}}(z)+\rho_{\mathrm{X}}(z)\right) g(z)-\nu \rho_{\mathrm{c}}^{0}}{1-\nu g(z)} .
$$

Even though the model could be solved from these equations, it is very convenient to find an effective equation of state approach to it, since this will allow us to better compare our model to alternative ones -e.g. quintessence scalar field models- and to confront it with the experimentally measured EOS. The procedure is thoroughly described in [14, 16]. The basic idea is that even a model with a non-conserved DE (like this one) can be represented by means of an "effective EOS" corresponding to a self-conserved dark energy. Thus we can contemplate the type II model under two different perspectives:

- In the original picture we see the model as it is: in this case, with self-conserved cosmon and matter-radiation densities together with variable cosmological parameters $G$ (Newton's coupling) and $\rho_{\Lambda}$ (vacuum energy density).

- In the DE picture we assume that the model (regardless of its real characteristics) has a constant $G$ and conserved DE and matter-radiation. The effective EOS arising from this picture is the one directly comparable to the experimental one.

The formula for $\omega_{\mathrm{e}}$ for type II models can be easily obtained from the condition that the DE is conserved in the DE picture, $\mathrm{d} \tilde{\rho}_{\mathrm{D}} / \mathrm{d} t+\alpha_{e} \tilde{\rho}_{\mathrm{D}} H=0$, where $\tilde{\rho}_{\mathrm{D}}$ is the DE density in this picture. It reads:

$$
\omega_{\mathrm{e}}^{(I I)}(z)=-1+\frac{1+z}{3 \tilde{\rho}_{\mathrm{D}}(z)} \frac{\mathrm{d} \tilde{\rho}_{\mathrm{D}}(z)}{\mathrm{d} z} .
$$


As the expansion history should not depend on the picture, we can match Hubble functions in each picture, hence: $G\left(\rho_{\mathrm{m}}+\rho_{\Lambda}+\rho_{\mathrm{X}}\right)=G_{0}\left(\rho_{\mathrm{m}}+\tilde{\rho}_{\mathrm{D}}\right)$. Substituting this equation in (31) and solving for $\mathrm{d} \tilde{\rho}_{\mathrm{D}}(z) / \mathrm{d} z$ we obtain the effective EOS parameter (37) of the type II $\Lambda$ XCDM model in a suitable form:

$$
\omega_{\mathrm{e}}^{(I I)}(z)=-1+\frac{\delta(z)}{3 \tilde{\Omega}_{\mathrm{D}}(z)},
$$

where

$$
\delta(z)=\alpha_{\mathrm{m}}\left(g(z) \Omega_{\mathrm{m}}^{0}-\tilde{\Omega}_{\mathrm{m}}^{0}\right)(1+z)^{\alpha_{\mathrm{m}}}+\alpha_{\mathrm{X}} g(z) \Omega_{\mathrm{X}}^{0}(1+z)^{\alpha_{\mathrm{X}}} .
$$

Note that the various $\Omega$ 's could depend (slightly) on the picture as they result from different fits to the same data, i.e. in general $\Delta \Omega_{\mathrm{m}}^{0} \equiv \Omega_{\mathrm{m}}^{0}-\tilde{\Omega}_{\mathrm{m}}^{0} \neq 0$. By requiring the same nucleosynthesis condition as in the previous model (i.e. $\left|r\left(z_{\mathrm{N}}\right)\right| \lesssim 10 \%$ ), we find that the only parameter constrained this time is $\nu$, which must satisfy the rather severe bound $|\nu| \lesssim 10^{-3}$ in order not to disturb nucleosynthesis predictions. Recall that for type I models the bound is on the parameter $\epsilon$ and is not so stringent, see Eq. (18).

\subsection{Numerical analysis of the EOS}

Although the coincidence problem can be solved similarly within the type II $\Lambda$ XCDM models [10], here we will just focus on the distinct behaviors of the effective EOS parameter (38), which are exemplified in Fig. 5a,b. We see that we can have transitions from quintessence into phantom regime (and vice versa), or just remain quintessence or phantom-like across the entire redshift interval relevant to SNIa observations. The model can even closely mimic a pure CC term while retaining its dynamical nature, which is revealed in its future behavior through the presence of a stopping point. In all these cases, the current value of $\omega_{\mathrm{e}}$ is close to -1 , hence compatible with the available data [2]. Finally, let us mention that in this model the behavior of $\omega_{\mathrm{e}}$ is modulated by the parameter $\Delta \Omega_{\mathrm{m}}^{0}$, which in this sense plays a similar role as $\nu$ in the type I models.

\section{Conclusions}

AXCDM models with local conservation of matter reveal themselves as generically capable to solve the cosmic coincidence problem, or at least to highly mitigate it. We have shown that irrespective of how the Bianchi identitity is implemented among the two density components $\left(\rho_{\Lambda}, \rho_{\mathrm{X}}\right)$ of the DE fluid, the overall behavior of the cosmological model is such that the ratio between the total DE and matter densities,

$r=\rho_{\mathrm{D}} / \rho_{\mathrm{m}}=\left(\rho_{\Lambda}+\rho_{\mathrm{X}}\right) / \rho_{\mathrm{m}}$, remains bounded throughout the entire history of the Universe, and can be of order one at present. This result is model independent, in the sense that we have not compromised the nature of the "cosmon" entity $X$ (in particular we did not tie it to a scalar field with some peculiar potential). We just used two independent ways of realizing the geometric Bianchi identity through the local conservation laws of the DE components, together with the local conservation of matter and a renormalization group inspired law for the running of the cosmological term. 

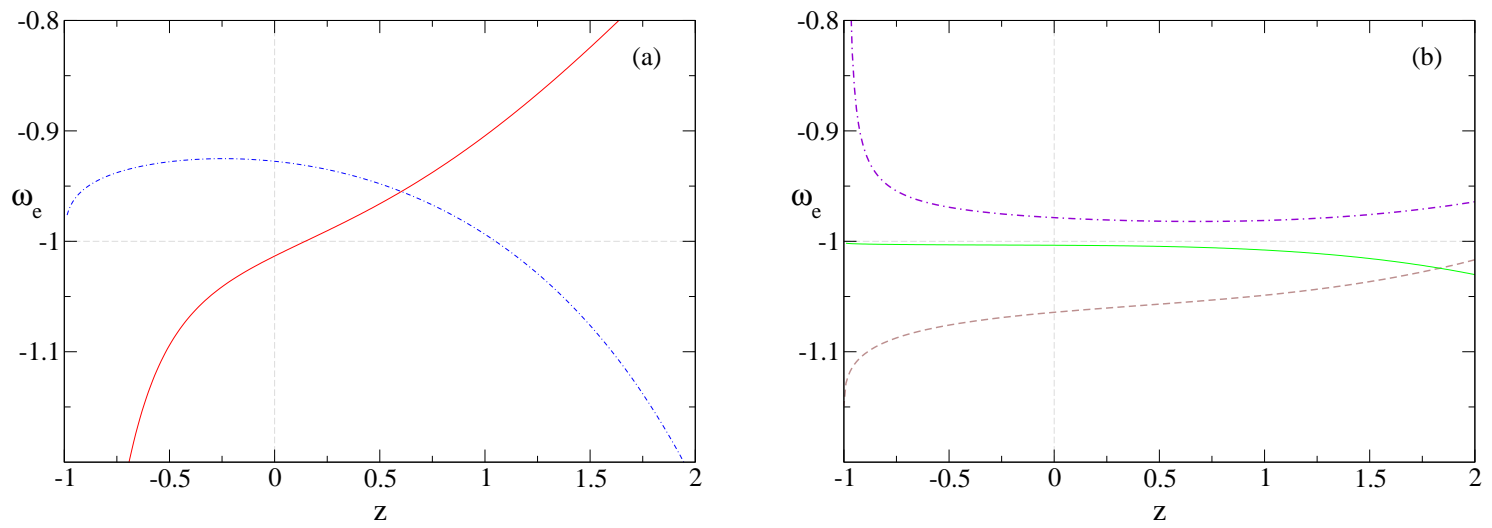

Figure 5. Some examples of the behavior of the effective EOS function for the type II cosmon models (38): (a) $\omega_{\mathrm{X}}=-1.65, \nu=+0.001, \Omega_{\Lambda}^{0}=0.67 \Delta \Omega_{\mathrm{m}}^{0}=0.01$ (solid line) and $\omega_{\mathrm{X}}=-0.85, \nu=-0.001, \Omega_{\Lambda}^{0}=0.3, \Delta \Omega_{\mathrm{m}}^{0}=-0.01$ (dash-dotted), illustrating the two types of possible transitions in the recent past; (b) $\omega_{\mathrm{X}}=-0.95, \nu=0.001$, $\Omega_{\Lambda}^{0}=0.75, \Delta \Omega_{\mathrm{m}}^{0}=0$ (solid line) and $\omega_{\mathrm{X}}=-1.15, \nu=-0.001, \Delta \Omega_{\mathrm{m}}^{0}=0$ and $\Omega_{\Lambda}^{0}=0.4$ (dashed) or $\Omega_{\Lambda}^{0}=0.8$ (dash-dotted), showing examples in which the EOS parameter mimics that of a $\mathrm{CC}$ or remains in the phantom or quintessence regime for all the redshifts attainable by present and scheduled supernovae experiments.

Acknowledgements. This work has been supported in part by MEC and FEDER under project 2004-04582-C02-01, and also by DURSI Generalitat de Catalunya under project 2005SGR00564. The work of HS is also financed by the Ministry of Science, Education and Sport of the Republic of Croatia.

\section{References}

[1] R.A. Knop et al., Astrophys. J. 598 (2003) 102; A.G. Riess et al., Astrophys. J. 607 (2004) 665.

[2] WMAP three year results: implications for cosmology, D.N. Spergel et al., astro-ph/0603449.

[3] S. Boughn and R. Crittenden, Nature 427 (2004) 45; New Astron. Rev. 49 (2005) 75.

[4] S. Dodelson, in: IRGAC 2006 (Barcelona, July 11-15 2006, http://www.ecm.ub.es/IRGAC2006/).

[5] M. Tegmark et al, Phys. Rev. D69 (2004)103501; S. Cole et al, Mon. Not. Roy. Astron. Soc. 362 (2005) 505.

[6] L. Amendola, Phys. Rev. D62 (2000) 043511.

[7] I.L. Shapiro and J. Solà, JHEP 0202 (2002) 006; Phys. Lett. B 475236.

[8] S. Weinberg, Rev. Mod. Phys 61 (1989) 1; V. Sahni, A. Starobinsky, Int. J. of Mod. Phys. A9 (2000) 373; Peebles, B. Ratra, Rev. Mod. Phys 75 (2003) 559; T. Padmanabhan, Phys. Rep. 380 (2003) 235; I.L. Shapiro and J. Solà, J. Phys. A (to appear), gr-qc/0611055.

[9] J. Grande, J. Solà and H. Štefančić, JCAP 0608 (2006) 011, gr-qc/0604057.

[10] J. Grande, J. Solà and H. Štefančić, Phys. Lett. B 645 (2007) 236, gr-qc/0609083

[11] R. R. Caldwell, M. Kamionkowski, N. N. Weinberg, Phys. Rev. Lett. 91 (2003) 071301.

[12] R. D. Peccei, J. Solà and C. Wetterich Phys. Lett. D195 (1987) 183.

[13] I.L. Shapiro, J. Solà, C. España-Bonet, P. Ruiz-Lapuente, Phys. Lett. 574 (2003) 149; JCAP 0402 (2004) 006; I.L. Shapiro, J. Solà, Nucl. Phys. Proc. Supp. 127 (2004) 71; I.L. Shapiro, J. Solà, H. Štefančić, JCAP 0501 (2005) 012.

[14] J. Solà, H. Štefančić, Phys. Lett. B624 (2005) 147.

[15] P. G. Ferreira and M. Joyce, Phys. Rev. D58 (1998) 023503.

[16] J. Solà and H. Štefančić, Mod. Phys. Lett. A21 (2006) 479; J. Phys. A 39 (2006) 6753. 